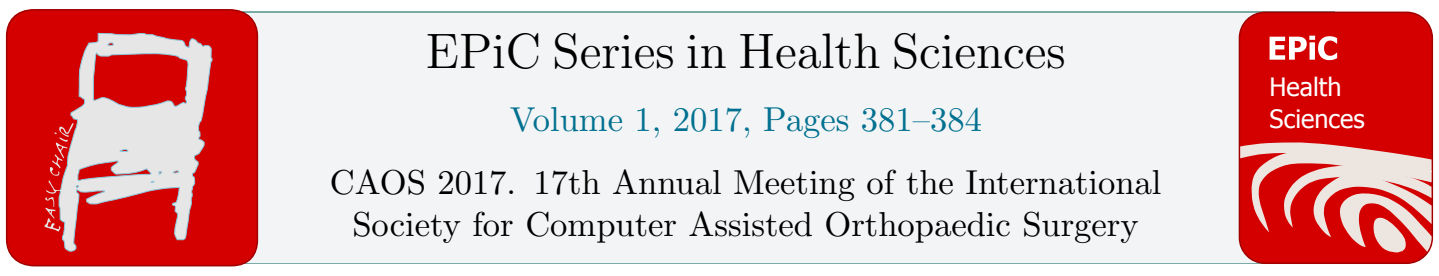

\title{
Patient-Specific Templates for Pedicle Screw Insertion in Spinal Fixation Surgery
}

\author{
Mohamed Fouda ${ }^{1}$, Ahmed Abdel Moghny ${ }^{2}$, Al-Othman A $^{3}$, Mohammed Ahmed Radwan ${ }^{4}$, Ahmed Ali \\ Toreih $^{4}$ and Mahmoud Hafez ${ }^{2}$ \\ ${ }^{1}$ Orthopedics and Traumatology Department, Ismailia General Hospital, Egypt \\ ${ }^{2}$ The Orthopaedic Department, October 6 University, Giza, Egypt \\ ${ }^{3}$ Department of Orthopedics, College of Medicine, University of Dammam, KSA \\ ${ }^{4}$ Faculty of Medicine, Suez Canal University, Egypt \\ Corresponding author: Mahmoud Hafez (․mhafez@,msn.com)
}

\section{Abstract}

The increased use of pedicle screws in scoliosis creates a challenge for accurate and safe placement of screw within the pedicle during the scoliosis surgery. Patient-specific templates (PST) is a novel method to guide the surgeons for allocating and detecting the positions and trajectories of pedicle screws in scoliosis surgery. Based on CT-scans and according to certain protocol, this technique will allow the surgeon to construct a 3D model of spine and present the stage and vertebrae which contain the most deformed bone. With preplanned surgery on specific software, we can develop an accurate and safe position of pedicle screws and its trajectories. This method has the ability to customize the placement and the size of each pedicle screw based on the unique morphology and landmarks of the vertebrae.

Introduction:

Computer-assisted orthopedic surgery (CAOS) based on medical imaging and software has helped in surgical setup with predictable outcome [1]. 3D printing also permitted rapid prototyping of real structures to allow the surgeon to simulate and evaluate the surgical steps and the postoperative

K. Radermacher and F. Rodriguez Y Baena (eds.), CAOS 2017 (EPiC Series in Health Sciences, vol. 1), pp. 381-384 
positioning and kinematics prior to the procedure. This technology has also been applied in joint replacements, complex fractures, plastic surgeries as well as maxilla-facial procedures as it allowed for the production of patient-specific surgical guides and prostheses [2].

Spinal surgery has benefitted from the use of screw-fixation techniques. Pedicle screws provide viable bone density to allow for safe anchorage and stability with the corresponding spine(s). In fact, their use has been quite difficult due to their variable size and direction in addition to the anatomical complexity of the surgical area. Several methods have been suggested for accurate insertion of pedicle screws such as fluoroscopy-guided and image-guided methods [3]. Navigation technique was found accurate but still expensive [4], while patient-specific templates (PST) could be used for precise insertion and fixation of the screws [5].

The aim of this work is to evaluate the outcomes of PST technique to for pedicle screw insertion and spinal fixation surgery.

\section{Methods:}

This technique relied on CT scans. The DICOM files were converted to 3D digital images to allow for segmentation and planning. Planning was done on Orthotaix which is proprietary software. 3D designing considered PST to rest on inferior articular process and lamina which secure a perfect contact and match with the vertebral surface. Two hollowed cylinders with diameter of $2.5 \mathrm{~mm}$ were used to direct the wire into the correct/accurate trajectories. The template was supported by two interior rods to secure the template during the drilling process.

The virtual design of the template was converted to stl files, which were transferred to 3D printer for digital fabrication. The printing technique was fused deposition melting (FDM) and the material used was polyamide nylon.

Results:

This technique showed its ability to customize the placement and the size of each screw based on the unique morphology and landmarks of the thoracic vertebrae. It is an easy and accurate method to allocate the pedicle screws.

Discussion:

Spine has complex anatomy and morphology. Scoliosis causes one or more variations such as kyphosis, wedging, vertebral rotation and deformed spondylolisthesis. Pedicle screw placement is a viable option for treating scoliosis but it presents some challenges to surgeons especially in patients with butterfly vertebrae and posterior fusion; and it is important to address these malformations prior to the surgical procedure. 3D CT scanning is beneficial in case of overlapping images in 2D images such as conventional X-ray films. PST has the advantage of designing patient-specific surgical plan to match the accurate screw size and entry point. It also helps in minimizing surgical errors, save 
operative time, reduce the intraoperative steps and relieve pain quickly as a minimally invasive procedure [6].

Conclusion:

PST can be used for placement and fixation of pedicle in scoliosis fixation surgeries with good applicability and high accuracy. The technique reduced the radiation exposure and operative time. The technique was capable of guiding the surgeons to the correct trajectories of pedicle screws.

Disclosure:

None.

\section{References:}

1. Lu S, Zhang YZ, Wang Z, Shi JH, Chen YB, Xu XM, et al. Accuracy and efficacy of thoracic pedicle screws in scoliosis with patient-specific drill template. Med Biol Eng Comput. 2012;50(7):751-758.

2. Müller A, Krishnan KG, Uhl E, Mast G. The application of rapid prototyping techniques in cranial reconstruction and preoperative planning in neurosurgery. J Craniofac Surg. 2003;14(6):899-914.

3. Rafael Cruz Bundoc, Giorgio De Guzman Delgado and Samuel Arsenio Munoz Grozman. A Novel Patient-Specific Drill Guide Template for Pedicle Screw Insertion into the Subaxial Cervical Spine Utilizing Stereolithographic Modelling: An In Vitro Study. Asian Spine J. 2017 Feb; 11(1): 4-14.

4. Intraoperative, full-rotation, three-dimensional image (O-arm)-based navigation system for cervical pedicle screw insertion. Ishikawa Y, Kanemura T, Yoshida G, Matsumoto A, Ito Z, Tauchi R, Muramoto A, Ohno S, Nishimura Y J Neurosurg Spine. 2011 Nov; 15(5):472-8.

5. Intraoperative, full-rotation, three-dimensional image (O-arm)-based navigation system for cervical pedicle screw insertion. Ishikawa Y, Kanemura T, Yoshida G, Matsumoto A, Ito Z, Tauchi R, Muramoto A, Ohno S, Nishimura Y J Neurosurg Spine. 2011 Nov; 15(5):472-8.

6. A novel computer-assisted drill guide template for thoracic pedicle screw placement: a cadaveric study. Ma T, Xu YQ, Cheng YB, Jiang MY, Xu XM, Xie L, Lu S Arch Orthop Trauma Surg. 2012 Jan; 132(1):65-72.

Figure 1: 3D planning of PST for pedicle screws. 

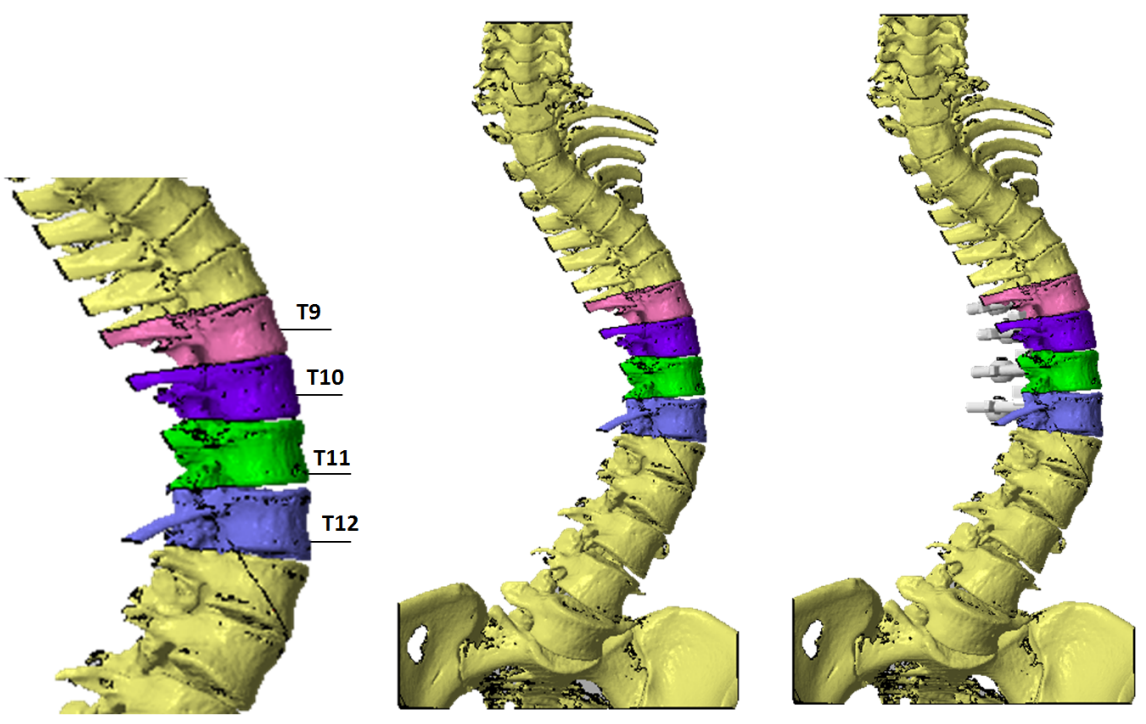

Figure 2: Virtual view of the PST and placement.
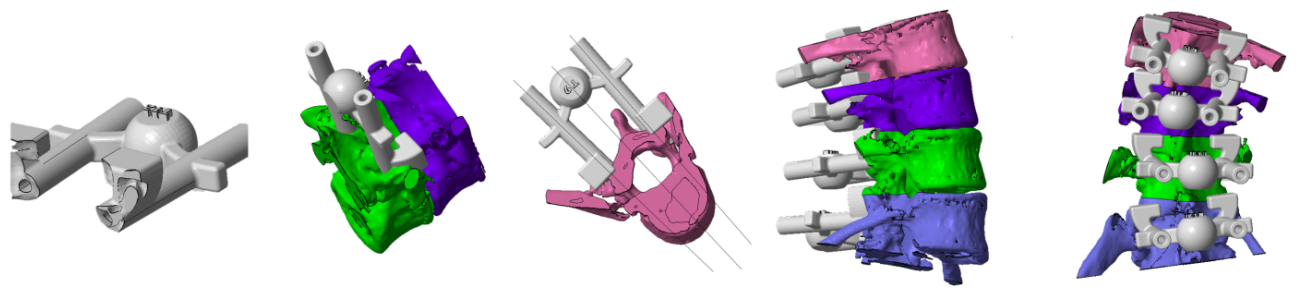